\title{
Revisando algunos aspectos de la educación bilingüe
}

\section{Josu Sierra}

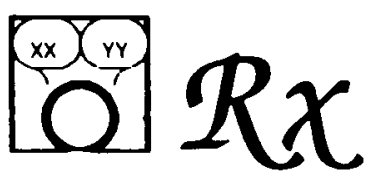

Tras más de una década de enseñanza bilingüe en que muchos niños españoles ban sido educados en dos o más de las lenguas del país, el autor revisa lo realizado y sus efectos y reflexiona sobre las consecuencias educativas a extraer. A este hecho bace propuestas didácticas concretas tanto en cuanto qué módulos de inmersión son viables como en cuanto a las actuaciones dentro de ellos.

Quisiera acometer en esta ocasión la reflexión sobre algunos puntos de la enseñanza bilingüe que están en fase de revisión y cuyas implicaciones prácticas son evidentes. Cuando digo enseñanza bilingüe me refiero, sobre todo, a aquella dirigida a sujetos cuya primera lengua es socialmente predominante y prestigiosa.

Han pasado ya más de diez años desde la implantación oficial de la educación bilingüe en la Comunidad Autónoma Vasca y, en mi opinión, ya va siendo hora de revisar algunos planteamientos de la misma, siempre basándonos en estudios y trabajos que están a nuestro alcance en este momento.

De un tiempo a esta parte van siendo numerosas las investigaciones acerca de las diferentes experiencias de enseñanza bilingüe que, tanto aquí como en el extranjero, se han llevado a cabo y a las que hemos podido acceder.

Partiendo de ellas, quisiera referirme a algunos «puntos calientes» que son objeto de nuestro interés y, de paso, analizar los programas vigentes en el País Vasco en esos mismos aspectos.

Por una parte, hemos podido conocer, hace no mucho, los resultados de nuestros dos programas de enseñanza bilingüe para castellanoparlantes, el B y el D (en el modelo B se imparte la enseñanza en euskera y castellano en una especie de inmersión parcial, mientras que en el $D$ la enseñanza es en euskera y el castellano constituye una asignatura). Estas evaluaciones recientes (Sierra, Olaziregi, 1989 y 1990) nos brindan la oportunidad de comparar algunos resultados con los obtenidos en trabajos realizados en otras partes del mundo.

Los puntos que tocaremos a lo largo del artículo serán los siguientes: 
En primer lugar, la importancia del tiempo de exposición en el proceso de aprendizaje de una segunda lengua, seguidamente pasaremos al papel de la interacción dentro de la clase, para continuar con una reflexión sore la L1 en diversos programas de inmersión y, finalmente, nos ocuparemos de la discusión en torno a la variable edad, con respecto al aprendizaje de la L2. Como colofón, analizaremos las consecuencias prácticas que debieran desprenderse de los puntos citados.

\section{LA CUESTION DEL TIEMPO DE EXPOSICION A LA L2}

En Canadá, algunos han denominado «superinmersión» al equivalente a nuestro modelo $\mathrm{D}$, es decir, al programa de educación en francés para francoparlantes, diferenciándolo de «early total inmersion», «partial inmersion», «delayed» o «late inmersion» por el hecho de ser más «intensivo» que cualquiera de ellos. Esta denominación de «superinmersión», sin embargo, no puede hacerse teniendo como referencia exclusiva la «intensividad», sino que debe tener en cuenta otras condiciones como las expresadas por Artigal (Artigal, 1989:18-22), concernientes a los postulados metodológicos a seguir en el proceso de aprendizaje de una segunda lengua.

Esta «superinmersión» entrecomillada es, por tanto, en Quebec la escuela francesa para francófonos a la que acceden algunos angloparlantes. Existen, pues, alumnos anglófonos escolarizados en este programa, a pesar de que lo común es que los sujetos anglófonos se escolaricen en programas «ingleses» o de inmersión. Esta situación se corresponde, con bastante exactitud, con la de los sujetos castellanoparlantes escolarizados en el modelo D en el País Vasco, si bien, quizá, los francófonos de Quebec sean en gran medida monolingües, cosa que no ocurre con los vascoparlantes.

Existen interesantes investigaciones acerca de los resultados obtenidos por los alumnos angloparlantes escolarizados en programas franceses y en programas de inmersión que, de paso, nos pueden resultar de utilidad en un análisis de nuestros modelos vigentes.

Por lo que respecta a la inmersión, hemos citado más arriba las diferentes opciones existentes en Canadá. Entre ellas, la más próxima al modelo «B oficial» del País Vasco sería la inmersión parcial. A pesar de ello, en la mayoría de las inmersiones parciales canadienses, el porcentaje de tiempo de enseñanza en L2 se sitúa desde el comienzo alrededor del 50 por ciento. En el modelo $B$, en cambio, cambia mucho (baja) esta proporción de Preescolar al Ciclo Inicial, para estabilizarse después en el 50 por ciento (ver gráfico en Sierra, 1991:53).

En cuanto a la inmersión total, puede equipararse con algunos modelos « $\mathrm{B}$ intensivos». Es decir, si en estos modelos B se hace un ciento por ciento de euskera en Preescolar y se trabaja la lectoescritura en euskera, podemos decir que es un $\mathrm{B}$ próximo a la inmersión, aunque habría que matizar en el terreno metodológico. Sin embargo, en la inmersión total el porcentaje de L2 desciende mucho a partir del quinto curso, cosa que no suele suceder tan pronunciadamente en los «B intensivos».

Las diferencias existentes entre los resultados de los modelos $\mathrm{B}$ y $\mathrm{D}$ se han puesto de manifiesto en más de una ocasión, pero considerando la variable «lengua materna», es decir, comparando sujetos de la misma L1, hemos de referirnos obligadamente al estudio EIFE-3 (Sierra, Olaziregi, 1990:37-41). 
Del citado trabajo pueden extraerse, entre otras, dos ideas importantes:

1.-Que los alumnos castellanoparlantes, tanto del modelo B como del D, obtienen el mismo nivel en las pruebas de castellano que los alumnos de programas no bilingües y que, por tanto, puede decirse que, aun la enseñanza en L2 más intensiva no produce en estos alumnos ninguna merma en su L1, extremo este corroborado en múltiples estudios, realizados con sujetos pertenecientes al grupo lingüísticamente mayoritario, en otras partes del mundo.

2.-Que, en general, los alumnos escolarizados en el modelo D obtienen mejores puntuaciones en euskera (L2) que aquellos que lo están en el modelo $\mathrm{B}$, aunque sea preciso matizar que, entre los sujetos del $\mathrm{B}$, se dan prácticamente todo tipo de puntuaciones. Incluso, algunos sujetos de este modelo, pocos, llegan al nivel de los alumnos del $\mathrm{D}$.

Es obvio pensar que estos últimos sujetos pertenecen a grupos «B intensivos», es decir, grupos cuyo porcentaje de enseñanza en L2 va de un 60 a un 80 por ciento.

Retomando el caso de los alumnos angloparlantes, citaremos la interesante investigación llevada a cabo en Canadá (Genesee, Holobow, Lambert, Chartrand, 1989), en la cual se comparan diversos tipos de inmersión:

Por un lado, el programa asimilable a nuestro D («superinmersión»), por otro, la inmersión total temprana y, finalmente, la inmersión parcial.

En esta investigación se aplicó a todos los alumnos una completa batería de pruebas, entre las que se incluían tests de lengua y matemáticas.

Las puntuaciones de francés obtenidas por los sujetos de inmersión total son equiparables a las logradas por los alumnos de «superinmersión», siendo para ambos grupos el francés la segunda lengua.

Por otra parte, ninguno de los dos grupos citados consigue el nivel de francés de los hablantes nativos del grupo de control. La principal diferencia respecto a los nativos se $\mathrm{da}$ en las destrezas orales.

A pesar de lo visto, se suponía que los sujetos del grupo de «superinmersión», gracias a su masiva exposición a la L2, obtendrían una clara diferencia a su favor en el nivel de francés con respecto al grupo de inmersión. Sin embargo, nada de esto ha ocurrido.

En opinión de los autores, en este tipo de programas, tanto de inmersión como más intensivos, y sin que medie una interacción significativa con los hablantes nativos, los alumnos llegarían a una especie de «tope» en su adquisición de la segunda lengua. Esta especie de «límite» sería muy difícil de superar con un simple aumento del tiempo de exposición dentro del programa.

Algunos investigadores atribuyen esta limitación a la metodología empleada por los profesores y de la que hablaremos más tarde. También conceden una gran importancia a la interacción con sujetos nativos para la superación de esa fase de meseta en la adquisición de la L2.

Genesee afirma, hablando de la cuestión del tiempo de exposición, que no existe una relación directa (Genesee, 1989:18) entre la cantidad de tiempo y los resultados obtenidos. Que el tiempo, en sí, no es una variable psicológica y, por tanto, no hay por qué creer que su sola presencia pueda determinar el proceso que se pone en marcha a la hora de aprender una lengua.

Volviendo a nuestras investigaciones, se han podido constatar estos extremos en estudios realizados aquí mismo. Concretamente, en un trabajo realizado con sujetos castellanoparlantes de los modelos B y D en $8 .^{\circ}$ de EGB (Olaziregi, Sierra, 1991). 
Se comparan en este estudio un grupo de sujetos pertenecientes a modelos «B oficiales» (con un porcentaje de euskera en torno al 50 por ciento), otro de sujetos escolarizados en $\ll B$ intensivos» (euskera en un 60-80 por ciento) y un grupo de sujetos castellanoparlantes del modelo $\mathrm{D}$. Todos ellos responden a unas características similares en cuanto a situación socioeconómica, entorno urbano, inteligencia y rendimiento escolar. Todos los sujetos pertenecían a $80^{\circ}$ de EGB y se les aplicaron pruebas tanto de euskera como de castellano. Solamente se midieron los aspectos lectoescritos.

Ateniéndonos a las puntuaciones de euskera, el modelo «B oficial» es el que obtiene, en este estudio, las puntuaciones más bajas. Entre el «B intensivo» y el «D castellanoparlante», sin embargo, no hay diferencia estadísticamente significativa, a pesar de que el tiempo de exposición al euskera es bastante superior en el modelo $\mathrm{D}$ (tanto los sujetos del modelo $\mathrm{B}$ como los del $\mathrm{D}$ pertenecen a zonas predominantemente castellanófonas).

Es preciso recalcar que no se midieron las destrezas orales, pero sabemos que en zonas donde la interacción con sujetos nativos es escasa, la utilización por parte de los alumnos de la L2 es más bien pasiva o reactiva y su nivel en ningún caso llega al de un hablante nativo, tal y como se refleja en los niveles de euskera de los castellanoparlantes del modelo $\mathrm{D}$ en $2 .^{\circ}$ de EGB, recogidos en el estudio EIFE-3.

Todo lo anteriormente dicho debiera ser suficiente para hacernos reflexionar acerca de la cuestión del tiempo de exposición o la cuestión de la «exposición pasiva» a la L2 y sus implicaciones en la aplicación concreta y diseño de los programas de enseñanza bilingüe.

Por un lado, ciertamente, es innegable su importancia, ya que sin un mínimo tiempo de exposición a la L2 no puede conseguirse un nivel suficiente en la misma. Un ejemplo de ello parecen ser los problemas de algunos programas de inmersión parcial (y de algunos modelos B) cuando los alumnos deben comprender textos complejos o expresarse por escrito en determinados temas.

Sin embargo, esto no significa que aumentando «enormemente» el tiempo de exposición los resultados mejorarán en la misma medida. El tiempo necesita de la concurrencia de otros factores que son los que realmente permiten obtener unos niveles de L2 más altos. La cantidad de input en L2 recibida por el alumno debe verse acompañada por otro tipo de variables que hacen este input aprovechable y eficaz.

En este sentido, la mitificación de programas de educación bilingüe con presencia masiva de la L2, como el D, que es en realidad un modelo pensado para sujetos vascohablantes de origen, pasando por alto aspectos más «cualitativos», corre el peligro de atribuir toda la eficacia al tiempo de exposición en L2 y olvidar la optimización del diseño, la metodología con que se aborda el proceso de adquisición/aprendizaje y la potenciación de estrategias comunicativas. Sobre todo ello abundaremos en el siguiente punto.

\section{PRODUCCION E INTERACCION: ¿QUIEN HABLA EN LA CLASE?}

Es fundamental en el aprendizaje de una segunda lengua el cómo se trabaja ésta, tanto en un aprendizaje «directo» como a través de cualquier materia del currículum.

El modo en que se trabaja en las aulas donde se enseña en L2 ha sido anali- 
zado por algunos investigadores, tanto en los casos en que el trabajo realizado se ha traducido en buenos resultados como en los que éstos no han sido tan positivos (Wong-Filmore, 1985:17-50).

El hecho de que el profesor sea «directivo» o «no directivo», que las clases sean «estructuradas» 0 «abiertas», las estrategias didácticas de los enseñantes, la participación de los alumnos, el tipo de lenguaje utilizado por el profesor en el aula al dirigirse a los alumnos en su L2... y un largo etcétera son elementos que marcan profundamente el proceso de su aprendizaje y, por ende, afectan a los resultados de modo determinante.

También Arnau (Arnau, Comet, Serra y Vila, 1992:42), citando diversos trabajos realizados en torno a este tema, dice que es la calidad de la enseñanza y su adaptación a las características de los alumnos la que determina la eficacia del modelo aplicado, afirmando que la elección de un determinado modelo de enseñanza bilingüe no garantiza un especial «grado de éxito».

Así pues, al hilo de lo anteriormente dicho, nos referiremos a algunas de las condiciones precisas para el logro de un suficiente nivel en L2.

Para Krashen era el «input comprensible» la variable fundamental en la adquisición de una segunda lengua (input hypotesis) (Krashen, 1981:100-137).

Para Swain, en cambio, aun siendo este input «conditio sine qua non", no es suficiente. Para ella también es imprescindible el «output comprensible» (Swain, 1985:252): el alumno debe tener opción para probar hipótesis y analizarlas en la lengua que está aprendiendo. «Del simple análisis semántico de la lengua, debe llegar a un análisis sintácticon y para ello, la clave es el output comprensible.

Desgraciadamente, dice Merrill Swain, el output comprensible no tiene sitio, generalmente, en nuestras aulas, ni siquiera en las de los programas bilingües.

De esta situación tampoco se salvan, por tanto, los programas de inmersión, ya que se ha comprobado en la realidad que los alumnos de estos programas, cuando llegan a un nivel determinado, sufren un parón en el desarrollo de su segunda lengua.

Es algo muy extendido en Canadá, el solicitar «programas de seguimiento» para los alumnos de programas de inmersión, con el fin de «rescatarles» de esa situación de estancamiento que se da en los cursos superiores, programas especiales para elevar su nivel en L2.

Esta situación citada de los cursos superiores parece ser en verdad preocupante (Cummins, 1987:202): algunos defectos y determinados errores se instalan permanentemente en la $\mathrm{L} 2$ de estos sujetos, convirtiéndose en incorregibles. Consecuentemente, esta segunda lengua se convierte en pobre, muchas veces no idiomática y falta de recursos, para desesperación de los profesores. La causa de estos problemas en el nivel de L2 tiene, pues, mucho que ver con la producción y la interacción en L2.

Según una investigación llevada a cabo en aulas de inmersión (Allen, Swain, Harley y Cummins, 1990:66; Genesee, 1991:16), en la cual se grabaron las interacciones que se daban dentro del aula durante las clases, la gran mayoría de las palabras que allí se escuchaban provenían del profesor. Cerca de un $\mathbf{4 0}$ por ciento de las expresiones que los alumnos de $3 .^{\circ}$ y $6^{\circ}$ cursos eran de una a dos palabras, mientras que las producciones de más de una oración no llegaban al 19 por ciento del total.

Esto indica claramente que los alumnos disponían de muy escasas ocasiones para poner en práctica esa segunda lengua que estaban aprendiendo, lo cual viene a confirmar la pesimista afirmación de Swain citada más arriba. 
Según observaciones realizadas en aulas de inmersión (Swain y Lapkin, 1989:153-157) se aprecia, en la metodología utilizada por los profesores, una enseñanza de la lengua basada más en el contenido de las materias que en la comunicación. La preocupación por el contenido a aprender en la segunda lengua no va, generalmente, acompañada por una planificación cuidada del lenguaje a utilizar.

De este modo, los principales problemas detectados se centran en:

a) Una incongruente corrección de errores del alumno por parte del profesor.

b) En la recepción por el alumno de un input funcionalmente restringido.

c) En la escasez de oportunidades que poseen los sujetos para usar la lengua que están aprendiendo, es decir, un output muy limitado.

Por tanto, está bastante claro que no es posible progresar gran cosa en el aprendizaje de una segunda lengua sin interacción y producción en la misma. Genesee propone al respecto un tipo de inmersión más centrado en el alumno, que Stevens denomina «Activity-centered immersion» (Genesee, 1987:73).

Pero esta interacción, ¿con quién y cómo puede hacerse del modo más conveniente? Es familiar para nosotros lo que Wong-Fillmore denomina recibir «junky input data» (Wong-Fillmore, 1985:25). Cuando la interacción entre los alumnos se da en aulas con pocos o ningún hablante nativo, los sujetos crean y desarrollan su propia «jerga», algo que a veces tiene poco que ver con la lengua real. En nuestro entorno se ha llamado a este fenómeno «euskera escolar», para denominar a esa L2 que se practica en algunas aulas, con mayoría castellanoparlante generalmente.

En un estudio realizado por Pellerin y Hammerly (Pellerin, Hammerly, 1986:592-605) se pone de manifiesto que, tras trece años de inmersión, la expresión oral de los sujetos está llena de incorrecciones «fosilizadas». Estos autores achacan el problema a la debilidad de los programas de inmersión para hacer frente a la corrección de errores y a la fosilización a causa de la exposición pasiva precoz a la $\mathrm{L} 2$, a la ausencia de una consistente enseñanza formal de la segunda lengua y al énfasis sobre el aspecto puramente comunicativo en detrimento de la exactitud y la corrección de la lengua.

Es cierto, al fin y al cabo, que son los alumnos quienes deben interaccionar, ya que el monólogo del profesor no hará, por sí solo, que desarrollen una competencia en L2. Sin embargo, la interacción que verdaderamente ayuda a aprender la segunda lengua es aquella que se lleva a cabo con hablantes nativos, y así se ha probado en numerosas evaluaciones con sujetos escolarizados en aulas de fuerte presencia nativa.

Desafortunadamente, en muchos casos, el profesor es el único hablante nativo (?) que el alumno tiene a su disposición, lo cual aumenta la responsabilidad del enseñante con respecto al alumno. Queda en su mano el marcar estrategias de participación, el programar y diseñar la segunda lengua en las diferentes actividades con el fin de ofrecer una progresión constante al alumno, ya que no sólo es preciso programar la materia a impartir, sino también la lengua que se va a utilizar para su aprendizaje. El profesor, por tanto, debe adaptar el diseño y la metodología del programa a las condiciones del entorno (presencia social de la L2, prestigio, procedencia de los alumnos, competencia del profesor, presencia de modelos nativos...) combinando intervención con potenciación del trabajo grupal, input comprensible con producción y reflexión acerca de la lengua con comunicación real. 


\section{EL NIVEL DE L1 EN LA INMERSION TOTAL Y PARCIAL}

Pasando a otro punto, digamos que desde los primeros momentos del modelo $\mathrm{B}$ se dio una gran importancia, en este programa dirigido a niños castellanoparlantes, al mantenimiento de un tiempo determinado para trabajar la lengua materna del alumno, es decir, el castellano. De esta manera, se argumentaba, el niño desarrollaría sin ningún inconveniente su L1 y al mismo tiempo se introduciría en el aprendizaje de la L2.

Del mismo modo, se quiso cuidar con atención especial la adquisición del cálculo y la lectura en la L1, con el convencimiento de que esto facilitaría la adquisición de tales destrezas por parte del alumno. Conscientemente, se rebajaba por tanto, en los comienzos del modelo B, la cantidad de input de euskera en los primeros años para así asegurar la L1 de los sujetos escolarizados en este programa. Esta introducción repentina de la L1 en un momento crítico del proceso de aprendizaje de la L2 no es algo inocuo sino que produce desajustes organizativos, complica el diseño y supone un freno en el aprendizaje de la L2.

Antes de que todo esto ocurriera, en Canadá iba muy adelantada la experiencia con programas de inmersión (Lambert, Tucker, 1972), pero estas experiencias no fueron tomadas como referencia en el diseño de los programas de enseñanza bilingüe en el País Vasco.

Entre los diferentes tipos de programa de inmersión, sin embargo, existe uno, la inmersión parcial, que dedica un 50 por ciento del tiempo lectivo a la L1 del sujeto.

A tenor de las diferentes evaluaciones realizadas en Quebec y el resto del Canadá, los sujetos escolarizados en programas de inmersión total obtienen mejores puntuaciones que aquellos pertenecientes a inmersión parcial, tanto en francés (L2) como en las diferentes asignaturas. Hamers y Blanc lo expresan rudamente cuando afirman que los sujetos de inmersión parcial tienen problemas, por ejemplo, en matemáticas y ciencias, como consecuencia de su escasa competencia en francés (Hamers, Blanc, 1983:319). También en la investigación llevada a cabo sobre los programas de inmersión en Ontario (Swain, Lapkin, 1983:63-69), efectivamente se constata que el insuficiente nivel de competencia en L2 de los alumnos de inmersión parcial y tardía tiene efectos negativos sobre los resultados académicos.

Por un lado, por tanto, esa limitada exposición a la L2 puede ser una fuente de problemas a la hora de cursar determinadas materias en L2. Pero, por otro lado, se podría argumentar que a cambio obtienen la ventaja de desarrollar mejor su lengua materna como consecuencia del diseño que en este sentido ofrece la inmersión parcial. Este argumento no se ha visto corroborado por las investigaciones realizadas.

Tal y como aparece en el estudio antes citado (Genesee, Holobow, Lambert y Chartrand, 1989), los sujetos de inmersión total, que por tanto no dedican un tiempo específico en los primeros cursos a su L1, son tan buenos como los sujetos de inmersión parcial en las pruebas de inglés (su L1) e incluso tan buenos como sus coetáneos de programas exclusivamente en inglés. Por tanto, el estudiar en programas como la inmersión total no produce a los sujetos ningún tipo de desventaja en su nivel de L1, tratándose de sujetos pertenecientes al grupo lingüísticamente predominante, en cuyo caso, un programa de mantenimiento de la L1 resulta suficiente para garantizar un nivel adecuado. Podemos preguntarnos, entonces, ¿dónde está la ventaja de la inmersión parcial? 
De los estundios realizados en el País Vasco, prede tambiém inferiirse la mis-

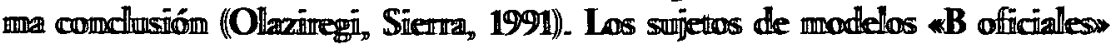
que dedicam un 50 por ciento del tiempo (excepto en preescolari) a trabajar em euslkera mo obtrienen mejones pumntuaciomes de castellano que los allummos de programas «B imtemsivosw o castell lamopparlantes escollarizados en el modelo D. Em las pruebras lectoescrittas de castefllamo mo aparece mimguma differencia estadistiicamemte sigmifficativa, mi siquiera com respecto al grupo de comirol del modelo A (emseñamza en castellamo).

Por el comtrario, en las pruebas de euskera los sujetos de los «B oficiallesw prumuturam por debajio de los ottros grupros citados.

Respecto all apremdizajie de la lectoescriturira, em un reciemte esturdio (Olaziregi, 1992-111-12) realizado em el País Vasco em el que se midió la comprensión lectora de sujetos castellamoparlantes escolarizados em modelos B y de Inmersióm (imo modelo D)), se comprureba que los allumnos de inmersióm puntúnam em la prueba de lecturra en castellano prácticamente al nivel de los alummos del modelo $B$, a pesar de que aprendierom a leer em euskera. Sin embargo, los sujetos del modelo B quedam bastante por debajo de los de inmersión en la prueba de lectura en euskera.

Esta relacióm entre lectura y mivel de segunda lengua es lo que Bossers analiza em un cuidadoso trabajo y concluye diciendo que solamente ocurre una transferencia directa de las habilidades lectioras en la $\mathrm{L} 1$ a la $\mathrm{L} 2$ cuando se ha adquirido un determinado nivel de esa $L .2$ (Bossers, 1991:56-57).

Esto explica que los alumnos de inmersión transfieran gran parte de la habilidad lectora inmediatamente a la L1 mientras que los alumnos del modelo $B$, que aprenden a leer en castellano, no pueden hacerlo a su $L 2$ porque no alcanzan ese nivel necesario en euskera. Paradójicamente, pues, estos sujetos del modelo $B$, en vez de trabajar en la $L 2$ para llegar a ese univel-umbralm, se han dedicado a aprender a leer y escribir en la L1, con lo que el resultado es el de un nivel más bajo de comprensión lectora en la $\mathbf{L} 2$.

No parece, consecuentemente, que los diseños tales como la inmersión parcial o el modelo B oficial estén consiguiendo los resultados esperados en $\mathbf{L} 2$. Por el contrario, parece claro que la L1 de los sujetos de inmersión total no corre ningún peligro, lo cual permite que consigan buenos niveles de $\mathrm{L} 2$ sin costo para su L1. Este hecho debe servir de acicate para una seria reflexión. Ha llegado, quizá, el momento de replantearse algunas cosas en este sentido en programas de baja intensidad. La cantidad de input que el sujeto debe recibir en $\mathbf{L} 2$ tiene que ser lo suficientemente elevada como para permitirle una progresión en su nivel de L2. Esto, no obstante, es compatible con un buen mantenimiento de su nivel de lengua materna.

No se justifica, por tanto, un cambio de lengua a la hora de acometer el aprendizaje de la lectoescritura o el cálculo, cambio que dificulta y frena la adquisición de la $L 2$, sin que ofrezca ventajas aparentes.

\section{LA EDAD, DE NUEVO, TEMA DE DISCUSION}

Existía la creencia, hasta hace bien poco bastante extendida, de que el mejor momento para aprender una segunda lengua era la más tierna infancia, y dentro de ella, el preescolar y primeros ciclos de la enseñanza, momentos que 
se debian aprovechar para aprender bien la L.2, ya que desprués este aprendizaje resultaría mucho más difficil.

Esta crecncia se basaba también, em gran medida, em las tesis acerca de la especial plasticidad del cerebro en estas etapas infantiles.

A este respecto, dicen Jacobs y Schumaman que:

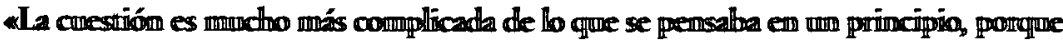
alhora se sabe que la plastrucidad del cercbro permamece durante toda la vidh dell orgamissmo y la batreralización y el "período crítico" mo son emtidades monolíricas (Jacobs, 1988). La suposición de Seliger de que cxisten múlthïples períodos criticos de adquisición del lempuaje tha encontrado recientemente base meurobiolópica en el Trabajo de

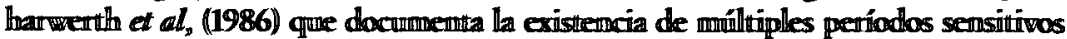
en el sistema visual..

Los estudios más recientes ponen en duda, en cierta medida, la absoluta ventaja atribuida a la uprecocidado (Genesee, 1987:56-57) y dan sentido a las posturas que preconizan la diversidad de opciones, momentos y medios por los que puede llegarse a la adquisición de una L2. Cuando se aprende una segunda lengua en un entorno escolar, con un gran nivel de abstracción y de un modo bastante descontextualizado, los alumnos emayoresn, al parecer, consiguen en menos tiempo un nivel equiparable al de los «pequeñoss. Esto es, al menos, lo que ha podido comprobarse al comparar la inmersión total precoz con la tardía (de dos cursos).

En investigaciones realizadas por Genesee y Adiv, en las cuales se evaluaron los niveles de francés una vez finalizadas las enseñanzas medias, no se encontraron diferencias entre los sujetos de inmersión total precoz y los de inmersión tardía, a pesar de que si contamos las horas de francés recibidas, los de inmersión tardía recibieron 1.400 frente a las 5.000 de los sujetos de inmersión precoz.

Para ver más claramente la influencia de la variable edad, se llevó a cabo otro tipo de evaluación. Los alumnos de inmersión precoz trabajaron en francés un ciento por ciento del tiempo en preescolar, $1 .^{\circ}$ y $2^{\circ}$. . Un 80 por ciento en $3 .^{\circ}$, un 60 en $4^{\circ}$ y un 40 entre $5 .^{\circ}$ y $8^{\circ}$ cursos.

Los de inmersión tardía, de 30 a 45 minutos diarios entre $1^{\circ}$ y 6. , solamente, y un 80 por ciento de francés en los cursos $7^{\circ}$ y $8 .^{\circ}$. De esta manera, estos últimos, al finalizar octavo, habían tenido una exposición equivalente a la de los sujetos de inmersión precoz hasta $2^{\circ}$ curso.

Utilizando una prueba normada por edades, los sujetos de inmersión tandía obtienen un nivel medio en $8^{\circ}$, mientras que los de inmersión precoz obtienen en la prueba de rendimiento en francés un nivel bajo en $2^{\circ}$ (debemos matizar, no obstante, que en otras inmersiones precoces más intensivas, como por ejemplo en los casos de Ottawa, no se ha repetido este fenómeno).

Estos resultados, por tanto, confirman la ventaja de los alumnos «mayores» en las situaciones citadas. Su mayor eficacia para aprender se basa, sin duda, en el mayor desarrollo cognitivo de estos sujetos (Swain, Lapkin, 1989:152). Esta eficacia, es verdad, se ha podido constatar con los aspectos más formales de la lengua solamente y, seguramente, en los procesos de aprendizaje en entornos no escolares se extraerán conclusiones diferentes y se obtendrán resultados de otro tipo.

A pesar de ello, la excesiva importancia que a veces se ha atribuido a la precocidad queda relativizada con los resultados de estas investigaciones.

Los diferentes aspectos de la lengua tienen su momento y su procedimiento más idóneo a lo largo del desarrollo y la evolución del niño. En este sentido, 
es lógico que, a medida que avanza el desarrollo cognitivo, exista una mayor facilidad para dominar los aspectos más cognitivos de la lengua. Otros componentes, en cambio, resultarán más difíciles con la edad, sobre todo los relacionados con los aspectos más informales de la comunicación, tales como la entonación, pronunciación, etc.

Lauren propone un programa ideal, para el aprendizaje de una segunda lengua, en dos fases: en la primera, muy precoz, los objetivos serían una buena pronunciación, una sintaxis básica automatizada y unas eficaces estrategias comunicativas. En una segunda fase, más tardía, se trabajarían aspectos de léxico y sintaxis en concordancia con el nivel cognitivo, más alto, de los sujetos mayores (Lauren, 1991:71).

Es posible aprovechar, así pues, la eficacia de los alumnos «mayores» y sería muy efectivo, por ejemplo, el crear «momentos intensivos» de aprendizaje de la L2 en los cursos superiores de la enseñanza. Esto resultaría especialmente práctico en el caso de los programas que obtienen pobres resultados de competencia en L2 como consecuencia de su diseño, por ejemplo, modelos A y algunos B en el País Vasco, ya que permitiría alcanzar niveles de L2 dignos a los sufridos alumnos abocados a quedarse siempre en una competencia incipiente, ya sea por el obsoleto diseño de aprendizaje como «asignatura de lengua», ya sea por la insuficiente intensidad y calidad del programa.

\section{CONCLUSIONES E IMPLICACIONES PRACTICAS}

Los puntos que hemos tocado no son, por supuesto, ni los más importantes ni los únicos que tiene planteados la enseñanza bilingüe hoy día, pero quieren ser un aviso acerca de la necesidad de revisión que nuestro sistema tiene en este momento.

Es urgente, en este sentido, investigar acerca de la calidad del input que reciben los alumnos castellanoparlantes de modelos $\mathrm{B}$ y $\mathrm{D}$, renovando los procedimientos y estrategias didácticas empleadas en el proceso de adquisición/ aprendizaje de la L.2. Esto urge en programas que, diseñados sobre la base de la lengua materna, tratan a alumnos de diferente $\mathrm{L} 1$ como iguales, poniendo muchas veces en peligro su desarrollo global (Arnau, Comet, Serra y Vila, 1992:100).

El pensar que el tiempo de exposición lo es todo no conducirá a la mejora de los problemas de calidad, funcionalidad y uso de la L2, cuestiones que luego tanto nos preocupan y verdaderas claves de este tipo de programas.

Algunos especialistas proponen, para un rápido y buen desarrollo de la L2, la construcción de un «entorno de aprendizaje óptimo», sugiriendo las siguientes actuaciones básicas (Ellis, 1985:161):

1.-Debe dirigirse al alumno una gran cantidad de input en L2.

2.-El alumno debe percibir la necesidad auténtica de comunicarse en la L2.

3.- Este debe poder controlar de algún modo o elegir el tema sobre el que se trata.

4.-Al menos al principio, es necesario atenerse al «aquí y ahora», evitando temas abstractos.

5.-El alumno debe tener la oportunidad de oír y producir diferentes formas para las diferentes funciones de la lengua-objetivo. 
6.-El alumno debe ser expuesto a gran cantidad de órdenes (mandatos).

7.-Debe, asimismo, disponer de abundantes oportunidades para completar, aclarar, confirmar, etc., sus expresiones.

8.-El alumno debe gozar de la posibilidad de prácticas desinhibidas, libres, de la L2, lo cual le dará la oportunidad de experimentar «nuevas» formas.

No estaría de más investigar acerca de la aproximación de estos principios básicos a nuestra realidad diaria en los programas bilingües.

Ya, de paso, también nos resultaría enriquecedor estudiar las estrategias metodológicas que utilizan los profesores y qué efecto producen en la comunicación dentro del aula.

Es hora de analizar la calidad de la L2 que son capaces de usar los castellanoparlantes de modelos D, sus incorrecciones fosilizadas, su capacidad comunicativa, etc., y tomar las medidas oportunas en cuanto a estrategias de corrección de errores, de aprendizaje formal de la lengua y de interacción con sujetos nativos.

Quizá también sea el momento adecuado para replantear los argumentos que se tuvieron en cuenta a la hora de diseñar el modelo $B$, sobre todo en cuanto al tratamiento de la $\mathrm{L} 1$ en los primeros cursos y el aprendizaje de la lectura, a la luz de los resultados actuales.

$Y$ finalicemos recordando que ahí está la inmersión tardía como opción muy válida para el proceso de aprendizaje de la segunda lengua en los cursos superiores para los programas de baja intensidad, necesitados también de una renovación metodológica.

Todo ello en el marco de una educación bilingüe que no puede detenerse, que debe responder a un entorno cada vez más exigente, donde la calidad será primordial, y la evaluación, constante, donde hemos de enfrentarnos ya al reto de la adquisición-aprendizaje eficaz de la Lengua Extranjera, situación que hará temblar algunas débiles estructuras de diseños mal planteados o deficientemente ejecutados, pero que puede representar el futuro de la enseñanza de las diversas lenguas de modo integrado y coordinado, algo que nos está haciendo mucha falta.

\section{Referencias}

Allen, P.; Swain, M.; Harley, B., y Cummins, J. (1990). «Aspects of classroom treatment: Toward a more comprebensive view of second language education»; en The development of second language proficiency. Cambridge University Press.

Arnau, J.; Comet, C.; Serra, J. M., y Vila, I. (1992). «La educación bilingüe». Barcelona: ICE/Horsori.

Artigal, J. M. (1989). "La inmersió a Catalunya». Ed. Eumo. Vic.

Bossers, B. (1991). "On thresholds, ceilings and short circuits: The relation between $L 1$ reading, $L 2$ reading and L2 knowledge», en J. H. Hulstijn, J. F. Matter (Eds.). «Reading in two languages». Amsterdam: Aila. Aila Review, 8.

Cummins. J. (1987). «Immersion programs: current issues and future directions». En Contemporary educationals issues: the canadian mosaic. Toronto: Copp Clark.

ElLIs, R. (1985). «Understanding second language acquisition». Oxford University Press.

Genesee, F.; Holobow, N. E.; Lambert, W. E., y Chartrand, L. (1989). 《Three elementary school alternatives for learning through a second language». En The modern language journal, 73.

Genesee. F. (1987). «Leaming through two languages». Newbury house. Cambridge. Mass. 1987.

GENESEE, F. (1989). «Second language learning in school settings: lessons from immersion». Conference on bilingualism, multiculturalism and second language learning. Ponencia. Esterel, Quebec.

GENESEE, F. (1991). «Second language leaming in school settings: lessons from immersion». Congreso de Aesla. Ponencia. Donostia. 
Hamers, J. F., y Blanc, M. (1983). «Bilingualité et bilinguisme». Pierre Mardaga Ed. Bruxelles.

Jacobs, F.; Schumann, J. (1992). Language acquisition and the neurosciences: towards a more integrative perspective», en Applied linguistics, 13, 3, 282-299.

KRASHEN, S. D. (1981). "Second language acquisition and second language learning». Oxoford: Pergamon Press.

Lambert, W.E., y Tucker G. R. (1972). «Bilingual education of children. The St. Lambert experiment». Newbury House. Rowley. Mass.

LAUREN, C. (1991). «A two-phase-didactics for school». En Joumal of multilingual and multicultural development, 12, 1991.

Olaziregi, I. (1992). "Evaluación de una experiencia de inmersión en el País Vasco». Ponencia XVII seminario «Llengues i Educació». Sitges (Barcelona). Octubre 1992.

Olaziregi, I., y Sierra. J. (1991). «Diversas alternativas de enseñanza bilingüe. Evaluación y comparación». Llengues i educació XVI seminari. Comunicación. Sitges. Barcelona.

Pellerin, M.; Hammerly, H. (1986). «L'expression orale apres treize ans d'immersion française». In The canadian modern language review, $42,3$.

Sierra, J. (1991). «La inmersión y la enseñanza bilingüe en el País Vasco». En Comunicación, lenguaje y educación, núm. 10. Madrid.

Sierra. J., y Olaziregi, I. (1989). «Eife-2. La enseñanza del euskera: La influencia de los factores». Servicio Central de Publicaciones. Vitoria-Gasteiz: Gobierno Vasco.

Sierra. J., y Olaziregi I. (1990) «Eife-3. La enseñanza del euskera: La influencia de los factores». Servicio Central de Publicaciones. Vitoria-Gasteiz: Gobierno Vasco.

SWAIN, M. (1985). "Communicative competence: some roles of comprebensible input and comprebensible output in its developments. En Input in second language acquisition. Ed. S. M. Gass y C. G. Madden. Newbury House. Rowley. Mass.

SwaIN, M., y LAPKIN S. (1989). «Canadian immersion and adult second language teaching: what's the connection». En The modem language joumal, 73, 1989.

SwaIN, M., y LAPKIN S. (1983). «Evaluating bilingual education: a canadian case study». Multilingual matters 2. Inglaterra: Clevedon.

Wong.Fillmorel, L. (1985). «When does teacher talk work as input?». En Input in second language acquisition. Ed. S. M. Gass y C. G. Madden. Newbury House. Rowley. Mass.

Revisando algunos aspectos de la educación bilingüe

Josu Sierra

CL\&E, 1993, 17, pp. 29-40

Resumen: El artículo revisa algunos «puntos calientes» de la educación bilingüe. En primer lugar se trata la importancia del tiempo de exposición en el proceso de aprendizaje de una segunda lengua. Seguidamente se pasa al papel de la interacción dentro del aula, para continuar con una reflexión crítica sobre la inmersión parcial y finalizar con la discusión en torno a la variable edad en el aprendizaje de la L2. En las conclusiones se aboga por determinados replanteamientos en el sistema de enseñanza bilingüe del País Vasco.

Datos sobre el autor: Josu Sierra es psicólogo y trabaja en el campo de la investigación sobre enseñanza bilingüe.

Dirección: Etxepare, 3. 48960 Galdakao (Bizkaia).

(C) De todos los artículos deberá solicitarse por escrito autorización de CL\&E y de los autores para el uso en forma de facsímil, fotocopia o cualquier otro medio de reproducción impresa. CL\&E se reserva el derecho de interponer las acciones legales necesarias en aquellos casos en que se contravenga la ley de derechos de autor. 